\title{
Effectiveness of a Custom-Made Temporary Obturator after Bilateral Total Maxillectomy
}

\author{
Kenji Nakamori $^{1}$, Manabu Yamagishi ${ }^{2}$, Keigo Takaya $^{2}$, Tomohiro Igarashi $^{1}$, Hiroyoshi Hiratsuka ${ }^{1}$ \\ ${ }^{1}$ Department of Oral Surgery, Sapporoy Medical University, Sapporo, Japan \\ ${ }^{2}$ Dental Laboratory Unit, Sapporoy Medical University Hospital, Sapporo, Japan \\ Email: nakamori@sapmed.ac.jp
}

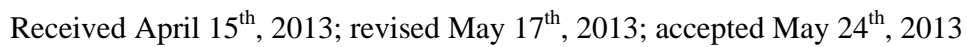

Copyright (c) 2013 Kenji Nakamori et al. This is an open access article distributed under the Creative Commons Attribution License, which permits unrestricted use, distribution, and reproduction in any medium, provided the original work is properly cited.

\begin{abstract}
Large oro-antral communication, associated with total maxillectomy, may lead speech and/or swallowing dysfunction. These surgical defects are packed with obuturator or prosthesis following surgery; however, achieving retention and stability is dependent on anatomical conditions. A 68-year-old Japanese female with large oro-antral communication arising because of bilateral total maxillectomy was referred for evaluating application of obturator. The temporary obturator was constructed as underlying $3 \mathrm{~mm}$ thick and covereing 1.5 - $2 \mathrm{~mm}$ thick ethylene vinyl acetate sheet (EVA). These two sheets formed the flexible hollow bulb portion, which aided retention and stability by engaging the undercut portion of the surgical defect. For the patients who have difficulty anatomical features for conventional prosthesis, this type of temporary obturator made with EVA sheets could be an effective solution in the early postoperative period.
\end{abstract}

Keywords: Custom-Made; Temporary Obturator; Bilateral Maxillectomy; Ethylene Vinyl Acetate

\section{Introduction}

Estimating Maxillary cancer is generally treated with surgical ablation, which often leaves large defects in the hard and soft palate. The resulting oro-antral communication generally causes speech and/or swallowing dysfunction. If immediate surgical reconstruction is not undertaken, the surgical defects are packed with gauze following surgery. However, the gauze packing needs to be changed frequently, so it is desirable to construct a hygienic and easily managed temporary obturator or prosthesis as soon as possible.

Achieving satisfactory retention and stability in the obturator prosthesis is dependent on anatomical conditions such as the height and contour of the residual alveolar ridge and the morphology of the defect site [1-3].

\section{Case Report}

A 68-year-old Japanese female with large oro-antrum communication arising because of bilateral total maxillectomy was referred to Department of Oral surgery of Sapporo Medical University Hospital for evaluating application of obturator. Twenty years prior to visit (in 1992), the patient had undergone right-side total maxillectomy combined with pre-plus post radiotherapy (50
Gy). Nineteen years after the right-side total maxillectomy (in 2011), another tumor aroused the left side of maxillary sinus, and patient had required total maxillectomy of left sides. After the both side of total maxillectomy, retaining only a narrow medial section of the soft and hard palate (Figure 1). One week after the operation, a stable, hygienic and flexible obturator was needed because the patient was experiencing severe trismus.

The temporary obturator was constructed as follows:

a) An impression was taken with elastomeric impresssion material to fabricate the working model.

b) Severe undercuts in the working model were blocked out with plaster.

c) A $3 \mathrm{~mm}$ thick sheet of ethylene vinyl acetate (EVA: Erkoflex ${ }^{\circledR}$ ERKODENT Erich Kopp GmbH, Pfalzgrafenweiler Germany) was heated and pressed to the model using a positive pressure method (Figure 2).

d) The margins of the EVA sheet were trimmed and another EVA sheet (1.5 - 2 mm thick Erkoflex ${ }^{\circledR}$ ) was adapted using a negative pressure method. The two EVA sheets adhered to each other because of their thermoplastic characteristics. Both sheets formed the hollow bulb portion of the obturator (Figure 3). The adhesion parts were trimmed and polished using silicon point. 
The hollow bulb obturator section aided retention and stability by engaging the undercut portion of the surgical defect. The elastic properties of EVA and the hollow bulb shape allowed the prosthesis to be easily inserted into the mouth in spite of the severe trismus. Speech and swallowing function was improved and the period of hospitalization was reduced using this temporary obturator (Figures 4 and 5).

\section{Discussion}

Maxillary cancer is generally treated surgery with or without radiotherapy. There were some reports of bilateral maxillary cancer, Shibuya et al. reported a remarkably high incidence of contralateral maxillary cancer (67 times higher) and suggested a link with radiotherapy [4]. In patients who have undergone bilateral maxillectomy, and whose anatomical features make it difficult for them to retain a conventional prosthesis, this type of temporary

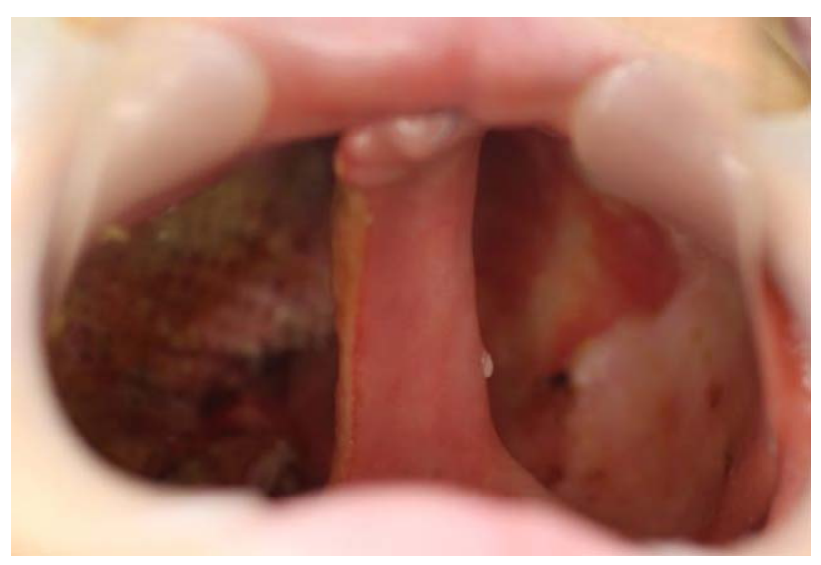

Figure 1. Intraoral findings. After maxillectomies on both sides (1992 left side and 2011 right side), only a narrow medial section of the soft and hard palate remained.

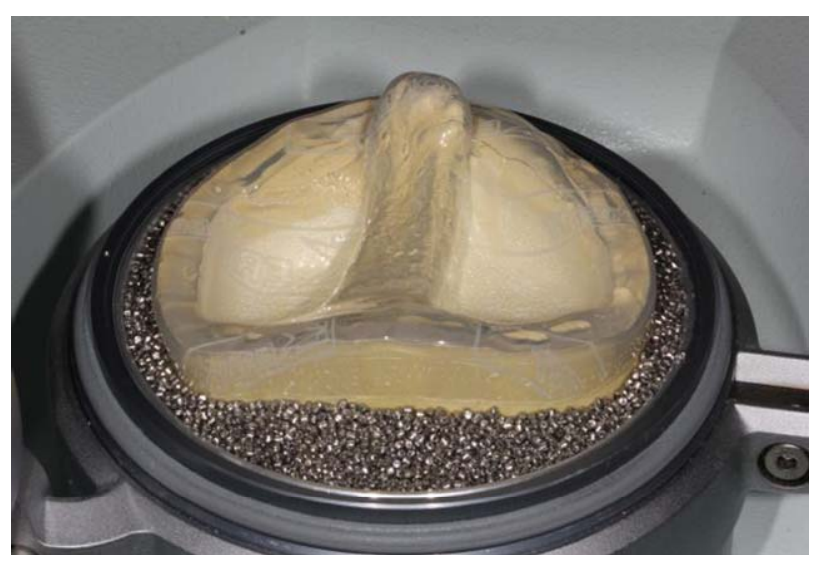

Figure 2. Fabrication of first layer. A working model was prepared by blocking excessive undercuts with plaster. The underlying $3 \mathrm{~mm}$ thick ethylene vinyl acetate (EVA) sheet $\left(\right.$ Erkoflex $^{\circledR}$ ) was formed using a positive pressure method.

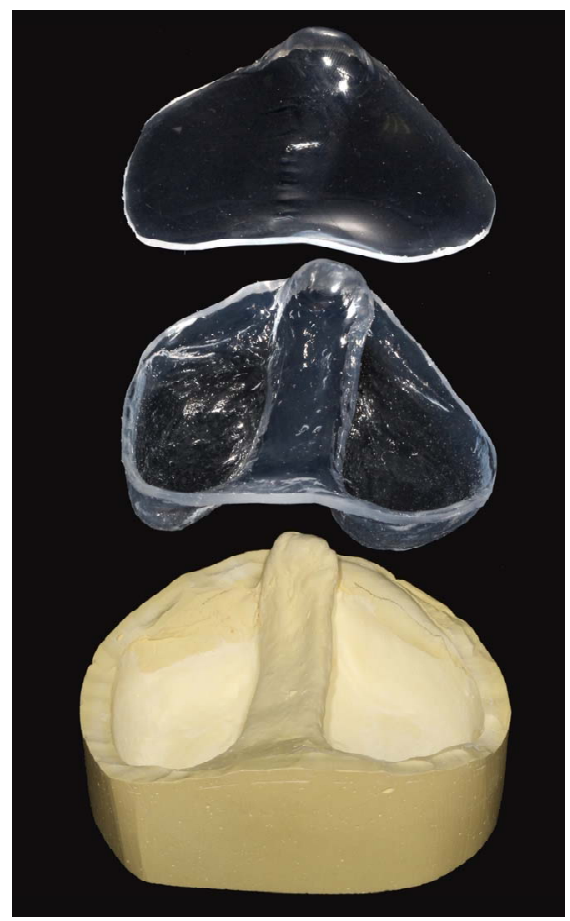

Figure 3. Fabrication of second layer. A secondary Erkoflex $^{\circledR}$ sheet (1.5 - 2 mm thick) was pressed using a negative pressure method.

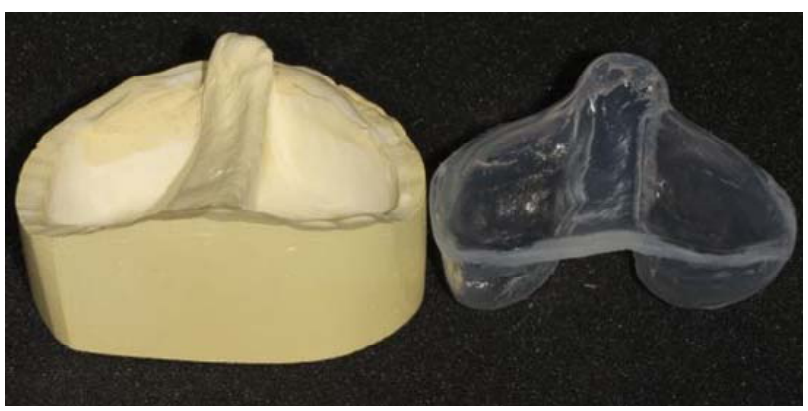

Figure 4. Custom-made temporary obturator. The hollow bulb and center parts are flexible and easy to insert into the mouth.

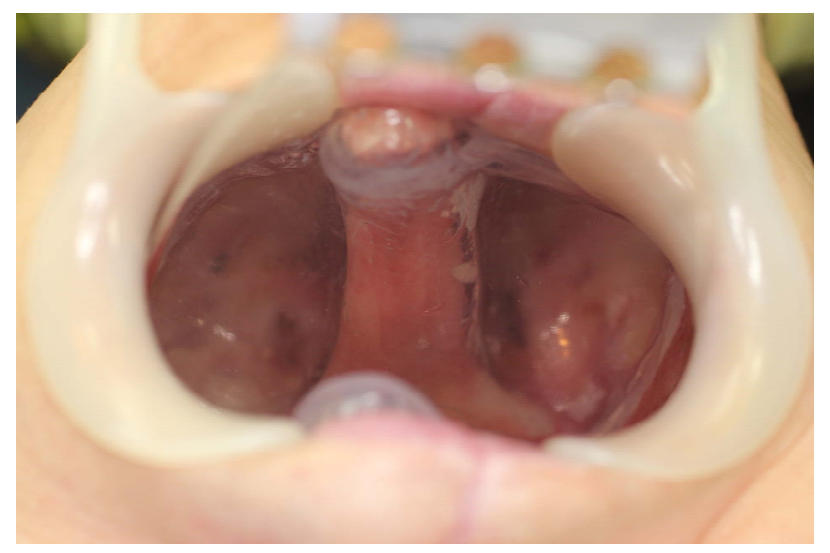

Figure 5. Intra-oral findings after insertion of the obturator. 
obturator made with EVA sheets could be an effective solution in the early postoperative period.

\section{REFERENCES}

[1] S. C. Ahila, K.V. Anitha and C. Thulasingam, "Comparison of Obturator Design for Acquired Maxillary Defect in Completely Edentulous Patients," Indian Journal of Dental Research, Vol. 22, No. 1, 2011, pp. 161-163.

doi:10.4103/0970-9290.79987

[2] F. Keyf, "Obturator Prostheses for Hemimaxillectomy Patients,” Journal of Oral Rehabilitation, Vol. 28, No. 9, 2001, pp. 821-829.

doi:10.1046/j.1365-2842.2001.00754.x
[3] H. Mukohyama, M, Haraguchi, Y. I. Sumita, H. Iida, Y. Hata, S. Kishimoto and H. Taniguchi, "Rehabilitation of a Bilateral Maxillectomy Patient with a Free Fibula Osteocutaneous Flap,” Journal of Oral Rehabilitation, Vol. 32, No. 7, 2005, pp. 541-544. doi:10.1111/j.1365-2842.2005.01463.x

[4] H. Shibuya, T. Amagasa, A. Hanai, J. Horiuchi and S. Suzuki, "Second Primary Carcinomas in Patients with Squamous Cell Carcinoma of the Maxillary Sinus," Cancer, Vol. 58, No. 5, 1986, pp. 1122-1125. doi:10.1002/1097-0142(19860901)58:5<1122::AID-CNC R2820580524>3.0.CO;2-4 